\title{
Postischemic Hyperglycemia Is Not Protective to the Neonatal Rat Brain
}

\author{
R. ANN SHELDON, J. COLIN PARTRIDGE, AND DONNA M. FERRIERO \\ Departments of Neurology [R. A. S., D. M. F.] and Pediatrics [J. C. P., D. M. F.], University of California San \\ Francisco, San Francisco General Hospital, San Francisco, California 94110
}

\begin{abstract}
Brain glucose concentration during and after hypoxia-ischemia may be one of the variables affecting outcome of asphyxial insults. Glucose given before global ischemic forebrain injury to adult rats increases morphologic brain damage, and postischemic insulin administration reduces selective neuronal necrosis and cortical infarction. Because glucose infusions are routinely used in the clinical management of perinatal asphyxia, we evaluated the role of glucose administration after ischemic neuronal damage to neonatal rat brain. Sprague-Dawley rat pups (postnatal d 7) were subjected to left common carotid artery ligation followed by $2.5 \mathrm{~h}$ of $8 \%$ oxygen (Levine procedure). The experimental group was subdivided so that pups received either systemic injections of glucose or saline immediately after the hypoxic insult. Animals were killed on postnatal d 12 and brain areas of ipsi- and contralateral cortex and caudate were calculated from camera lucida tracings. There was no significant difference in size of brain infarction between postischemic glucose-treated and postischemic saline-treated pups. However, hypoxic-ischemic brains did show more severe neuronal damage when hyperglycemia was induced after asphyxia. Because postischemic hyperglycemia does not attenuate and may exacerbate injury, we recommend careful monitoring of blood glucose so that hyperglycemia does not occur during resuscitation of asphyxiated infants. (Pediatr Res 32: 489-493, 1992)
\end{abstract}

\section{Abbreviations}

NADPH-d, NADPH diaphorase

EAA, excitatory amino acid

Neurologic morbidity occurs in $20-30 \%$ of infants suffering acute perinatal asphyxia in the United States (1). The hypoxicischemic encephalopathy seen in survivors of perinatal asphyxia is a commonly encountered clinical problem and is thought to be the largest contributor to static encephalopathies in infants and children (2). Hypoglycemia is a frequent metabolic derangement among asphyxiated infants and has additive deleterious effects on the CNS sequelae of perinatal asphyxia (3-5). Standard resuscitation procedures use glucose infusions to correct posthypoxic hypoglycemia, but this procedure often results in hyperglycemia (6).

Received February 24, 1992; accepted May 28, 1992.

Correspondence: Donna M. Ferriero, M.D., Neurology and Pediatric Services, San Francisco General Hospital, University of California-San Francisco, San Francisco. CA 94110

Reprint requests: Dr. Ferriero, Neurology Service 4M62, San Francisco General Hospital, 1001 Potrero Ave., San Francisco, CA 94110.

Supported by grants from the University of California San Francisco Academic Senate Committee on Research, Pre-Tenure Award, and Research Evaluation and Allocation Committee to D.M.F.
The brain is predominantly glucose-utilizing organ, but it is capable of using alternative substrates such as acetoacetate and $\beta$-hydroxybutyrate (7). These alternate fuels may ameliorate the neuropathologic effects of hypoglycemia under circumstances in which oxygen delivery is adequate to allow oxidation to energyyielding substrates. During hypoxia or ischemia, brain glucose supplies are depleted. Glucose and alternate fuel delivery become compromised, thereby exacerbating neuronal damage (7). Thus, brain glucose concentration during and after ischemia may be one of several variables affecting outcome after hypoxic-ischemic insults.

The effect of glucose on hypoxic-ischemic brain injury has been studied in both newborn and adult animal models with conflicting results. The majority of the data in the mature brain support a deleterious effect of hyperglycemia, with elevated blood glucose concentrations associated with worsened neurologic outcome $(8,9)$. Increased pathologic damage, cerebral edema with impaired cerebral perfusion, and slowed recovery of normal brain metabolic processes occur $(10,11)$. Studies on neonatal animals are contradictory (12-14). Some investigators have found beneficial effects of glucose administered immediately after the hypoxic-ischemic insult but no substantial protection when glucose treatment is delayed by $1 \mathrm{~h}(14)$. Other investigators have found no difference in the degree of cerebral damage in asphyxiated hyperglycemic animals compared with normoglycemic counterparts (12).

It is clinically important to understand the role of hyperglycemia after hypoxic-ischemic brain damage because hyperglycemia is a common metabolic complication of term and premature infants undergoing resuscitation in the neonatal period. Current resuscitation techniques involve the administration of glucose to asphyxiated infants during the initial and recovery periods after perinatal asphyxia and could thus have deleterious effects on subsequent neurologic outcome or survival. To explore the effects of hyperglycemia on hypoxic-ischemic neonatal brains, we treated neonatal rat pups with glucose immediately after hypoxic-ischemic injury.

\section{MATERIALS AND METHODS}

We used a model of hypoxia-ischemia based on the Levine procedure (15-17). Sprague-Dawley litters of 7-d-old rat pups were culled to 10 pups each. Animals were divided into an ischemic group (six pups) and a nonsurgical control group (four pups). All animal research was approved by the University of California San Francisco Committee on Animal Research and was performed with the highest standards of humane care as set forth in the Guide for the Care and Use of Laboratory Animals, U.S. Department of Health and Human Services, Publication Number 85-23, 1985.

The six pups from each litter designated for the Levine procedure were anesthetized with $1 \%$ halothane in $40 \%$ oxygen, balance nitrogen, and the left carotid artery was exposed and permanently ligated. The incision was sutured and the pups were 
returned to the dam for a $2-h$ recovery and feeding period. These pups were placed in containers through which humidified $8 \%$ oxygen and balance nitrogen flowed for the next $2-1 / 2 \mathrm{~h}$. The containers were partially submerged in a $37^{\circ} \mathrm{C}$ water bath, and the body temperature of one animal was monitored by external probe throughout the hypoxic session. Body temperature was maintained in the $36-37^{\circ} \mathrm{C}$ range by adjusting the water bath temperature. Upon removal from the hypoxic containers, three of these pups were given an intraperitoneal injection of $0.1 \mathrm{~mL}$ $50 \%$ glucose $(2.5 \mathrm{~g} / \mathrm{kg})$ and the remaining three pups were given $0.1 \mathrm{~mL}$ of normal saline by the same route. The tail vein blood glucose level of each animal was obtained $5 \mathrm{~min}$ after glucose or saline injection. The whole blood glucose levels were monitored using glucose reagent strips read on a glucometer (Ames Co., IN). The total number of animals constituting the saline-treated ischemic group was 17 , and the number in glucose-treated ischemic group was 19.

The four remaining animals from each litter served as nonsurgical controls. They were removed from the dam with the surgically treated pups so that feeding would be concordant for the two groups. Three of these received $0.1 \mathrm{~mL} 50 \%$ glucose injections $(n=17)$ equivalent to the surgical animals, and the remaining pup received an injection of $0.1 \mathrm{~mL}$ normal saline.

Five d after hypoxic-ischemic injury, all pups were anesthetized with $50 \mathrm{mg} / \mathrm{kg}$ pentobarbital intraperitoneally and were then perfused through the ascending aorta with cold $4 \%$ paraformaldehyde in $0.1 \mathrm{M}$ phosphate buffer ( $\mathrm{pH} 7.4$ ). Brains were then removed and immersed in the same fixative for $4 \mathrm{~h}$, then transferred to ice-cold $30 \%$ sucrose in $0.1 \mathrm{M}$ phosphate buffer. Forebrain sections were cut at $50-\mu \mathrm{m}$ intervals using a vibratome. Alternate sections were stained with cresyl violet or prepared for NADPH-d histochemistry by placing fixed sections in wells containing incubation medium $[0.1 \mathrm{M}$ Tris- $\mathrm{HCl}(\mathrm{pH} 8), 0.2 \mathrm{mM}$ NADP, $15 \mathrm{mM}$ sodium malate, $0.2 \mathrm{mM}$ nitro blue tetrazolium, $1 \mathrm{mM} \mathrm{MnCl}$, and $0.1 \%$ Triton-X] for $2 \mathrm{~h}$ at $37^{\circ} \mathrm{C}$. All sections were mounted onto gelatinized slides, dehydrated, and coverslipped with Depex (Biomedical Specialties, St. Monica, CA) (17).

Brain infarction was determined by calculating mean brain areas of surviving tissue from camera lucida drawings of three serial sections from each brain at the level of the anterior commissure. The areas of left (injured or ipsilateral) cortex and caudate and right (noninjured or contralateral) cortex and caudate were calculated using the Video Image Analysis System (Ted Pella Inc., Tustin, CA). The results are expressed in $\mathrm{mm}^{2}$ for the remaining tissue (Table 1 ).

To determine the degree of cellular neuronal damage, cresyl violet Nissl stain and NADPH-d histochemistry were used. We have previously shown that the subpopulation of neurons containing NADPH-d is selectively resistant to hypoxic-ischemic injury in the neonatal rat and that these neurons therefore serve as markers for this type of injury (17). Light microscopic damage was rated such that $0=$ no detectable neuronal cell loss or gliosis, $1=$ columnar damage in the cortex involving predominantly layers II through IV with selective sparing of the NADPH-d reactive neurons, and $2=$ contiguous areas of gliosis with resulting neuronal cell loss including NADPH-d reactive cells through all layers of the cortex. Brains were scored by an observer blinded to the postischemic treatment.
Statistical analysis was performed by unpaired $t$ test and oneway analysis of variance with Scheffe's test for post hoc comparison for parametric data. The Wilcoxon signed rank test was used for nonparametric analysis of categorical data.

\section{RESULTS}

Animals receiving saline or glucose injections without hypoxiaischemia showed no histopathologic damage (Fig. $1 A$ ). Blood glucose concentrations of saline-injected animals ranged from 20 to $40 \mathrm{mg} / \mathrm{dL}(1-2 \mathrm{mM})$, and those of the glucose-injected animals ranged from 200 to $240 \mathrm{mg} / \mathrm{dL}(11-13 \mathrm{mM})$. The Levine procedure produced lesions of the frontoparietal cortex and dorsolateral caudate as previously described (Fig. 1B) (17); glucose posttreatment exacerbated the degree of infarction (Fig. $1 C$ ).

Brain infarction. There was a significant difference between the area of remaining ipsilateral cortex and the area of the contralateral cortex in ischemic brains with saline $(14.63 \pm 1.90$ versus $\left.20.50 \pm 0.61 \mathrm{~mm}^{2} ; p<0.05\right)$ as well as glucose posttreatment $\left(12.79 \pm 2.09\right.$ versus $\left.21.11 \pm 0.83 \mathrm{~mm}^{2} ; p<0.001\right)$. The contralateral area was the same as cortical area in nonischemic brains; therefore, the contralateral areas were used to compare the effect of posthypoxic treatment on the degree of damage (Table 1). There was a significant difference between ipsilateral and contralateral caudate in glucose-treated ischemic pups (5.69 \pm 2.60 versus $\left.8.09 \pm 1.52 \mathrm{~mm}^{2} ; p<0.01\right)$ and saline-treated $\left(5.97 \pm 2.71\right.$ versus $\left.8.09 \pm 1.52 \mathrm{~mm}^{2} ; p<0.001\right)$ ischemic caudate (Table 1). When areas are expressed as percentages (ipsilateral over contralateral) to minimize the effect of individual variation brain size, the same posttreatment effect is again demonstrated (Fig. 2). However, there was no significant difference between glucose-treated and saline-treated infarct areas.

Neuronal cell loss. Although the area of ipsilateral ischemic brain infarction in glucose-treated and saline-treated animals was not significantly different, cellular damage was more severe after hypoxia-ischemia when animals received glucose $(p=0.03)$. Only $5 \%$ of glucose-treated and $18 \%$ of saline-treated animals showed columnar or discrete damage to the cortex, whereas $53 \%$ of glucose-treated and $29 \%$ of saline-treated animals demonstrated continuous areas of gliosis and neuronal loss through cortex and/or caudate (Fig. 3). Nissl-stained sections through infarcted cortex of normoglycemic brains showed a columnar pattern of cell loss (Fig. 4B), whereas hyperglycemic ischemic brains showed more extensive neuronal damage through all layers of the cortex as well as gliosis of the white matter (Fig. $4 C$ ). Brains with a microscopic score of 2 exhibited necrosis of neurons throughout all layers of the cortex and germinal matrix as well as destruction of axons in the corpus callosum and striatal perikarya.

NADPH-d histochemistry in normoglycemic ischemic brains showed preservation of the NADPH-d reactive cells around areas of columnar loss as previously reported (17) (Fig. $4 E$ ). However, the hyperglycemic ischemic brains showed loss of these cells with only rare degenerating cells remaining in the penumbra zone (Fig. $4 F$ ).

Table 1. Brain areas of injured and control pups*

\begin{tabular}{|c|c|c|c|c|c|c|}
\hline & \multicolumn{2}{|c|}{ Saline and hypoxia-ischemia } & \multicolumn{2}{|c|}{ Glucose and hypoxia-ischemia } & \multicolumn{2}{|c|}{ Control } \\
\hline & Left & Right & Left & Right & Left & Right \\
\hline Cortex & $14.63 \pm 1.90+$ & $20.50 \pm 0.61$ & $12.79 \pm 2.09 \ddagger$ & $21.11 \pm 0.83$ & $21.00 \pm 0.61$ & $20.40 \pm 0.73$ \\
\hline Caudate & $5.97 \pm 2.71 \$$ & $8.09 \pm 1.52$ & $5.69 \pm 2.60 \ddagger$ & $8.10 \pm 1.44$ & $7.96 \pm 1.28$ & $8.20 \pm 1.68$ \\
\hline
\end{tabular}

* Data is expressed as mean $\pm \mathrm{SEM}$ in $\mathrm{mm}^{2}$.

$+p<0.05$.

$\ddagger p<0.001$.

$\$ p<0.01$. 

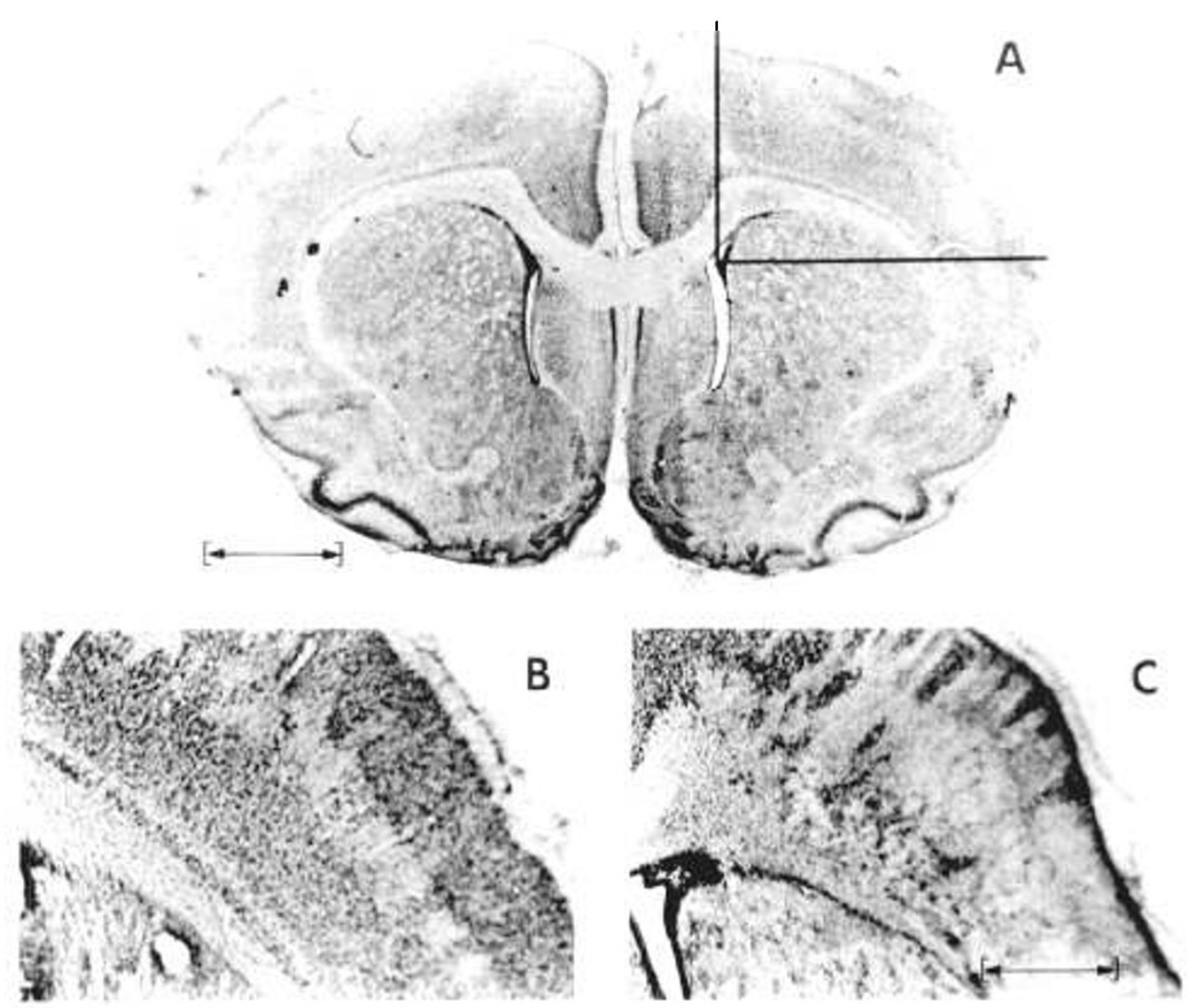

Fig. 1. $A$, Nissl-stained coronal section through anterior forebrain of nonischemic neonatal rat brain treated with glucose. $B o x$ indicates comparable areas magnified in $B$ and $C$. Scale $=2 \mathrm{~mm}$. B. Saline-treated ischemic brain section through comparable area indicated in $A$ showing columnar pattern of cell loss in the deep layers of cortex. $C$. Glucose-treated ischemic brain showing massive neuronal cell loss through cortex and caudate despite preservation of outer cortical architecture. Scale $=100 \mu \mathrm{m}$.

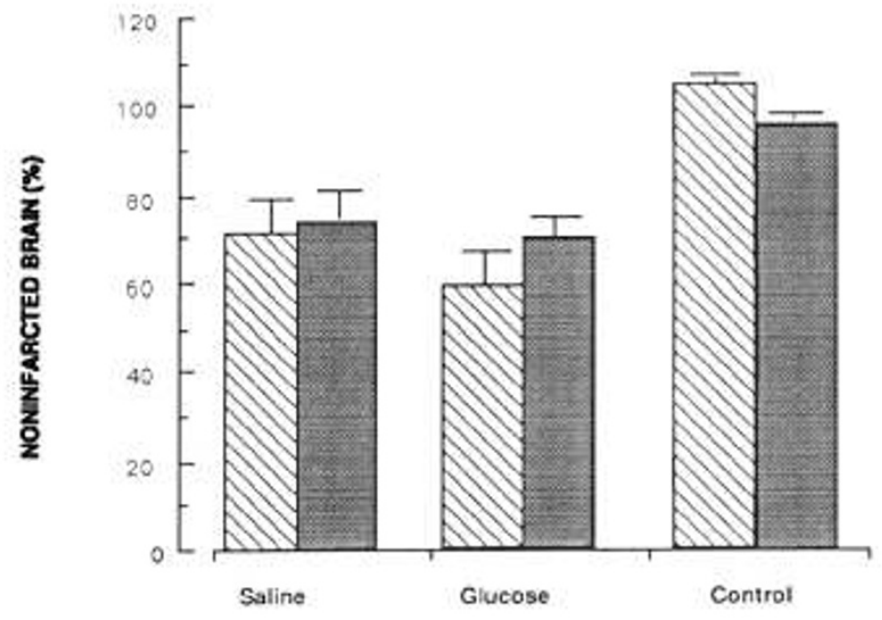

Fig. 2. Percentage of cortex $(\mathbb{Q})$ and caudate $(\square)$ remaining (ipsilatcral/contralateral $\times 100 \%)$ after hypoxic-ischemic insult in glucosetreated and saline-treated animals.

\section{DISCUSSION}

We have found that there is significant damage to the ischemic neonatal brain in the presence of postinjury hyperglycemia. Damage to neurons is more severe than that seen in ischemic brains of normoglycemic animals. Using the same model of focal hypoxia-ischemia and preinsult hyperglycemia in the $200-300$ mg\% range, other studies have shown no significant effect of pretreatment with glucose on subsequent cerebral injury after hypoxia-ischemia (12). In this study, we investigated the role of hyperglycemia after hypoxic-ischemic insult. Our data in rat

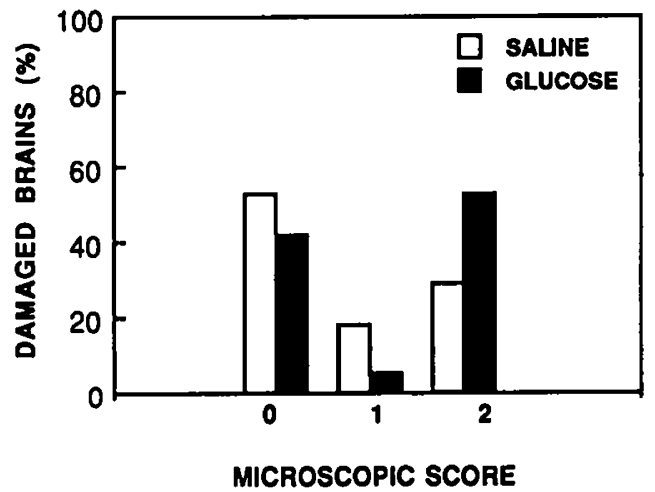

Fig. 3. Percentage of hypoxic-ischemic brains, either saline- or glucose-treated, categorized by degree of damage demonstrated microscopically. Ischemic brains from animals treated with glucose showed more extensive damage microscopically $(p=0.03)$.

pups demonstrates a potentially detrimental effect of glucose administration after hypoxia-ischemia in the immature brain.

This deleterious effect contrasts with the protective effect of immediate postinjury glucose administration in the neonatal rat shown by Hattori and Wasterlain (14). Their model differed from our focal ischemia model in that global hypoxia-ischemia was induced by bilateral carotid ligation followed by $1 \mathrm{~h}$ of hypoxia. They found that glucose given immediately after hypoxia reduced neocortical infarct volume to $37 \%$ of that in unsupplemented animals, but glucose treatment $1 \mathrm{~h}$ after the end of hypoxic exposure was not protective against infarction. However, the damage seen in global models of hypoxia-ischemia such as the bilateral carotid ligation model is much more exten- 

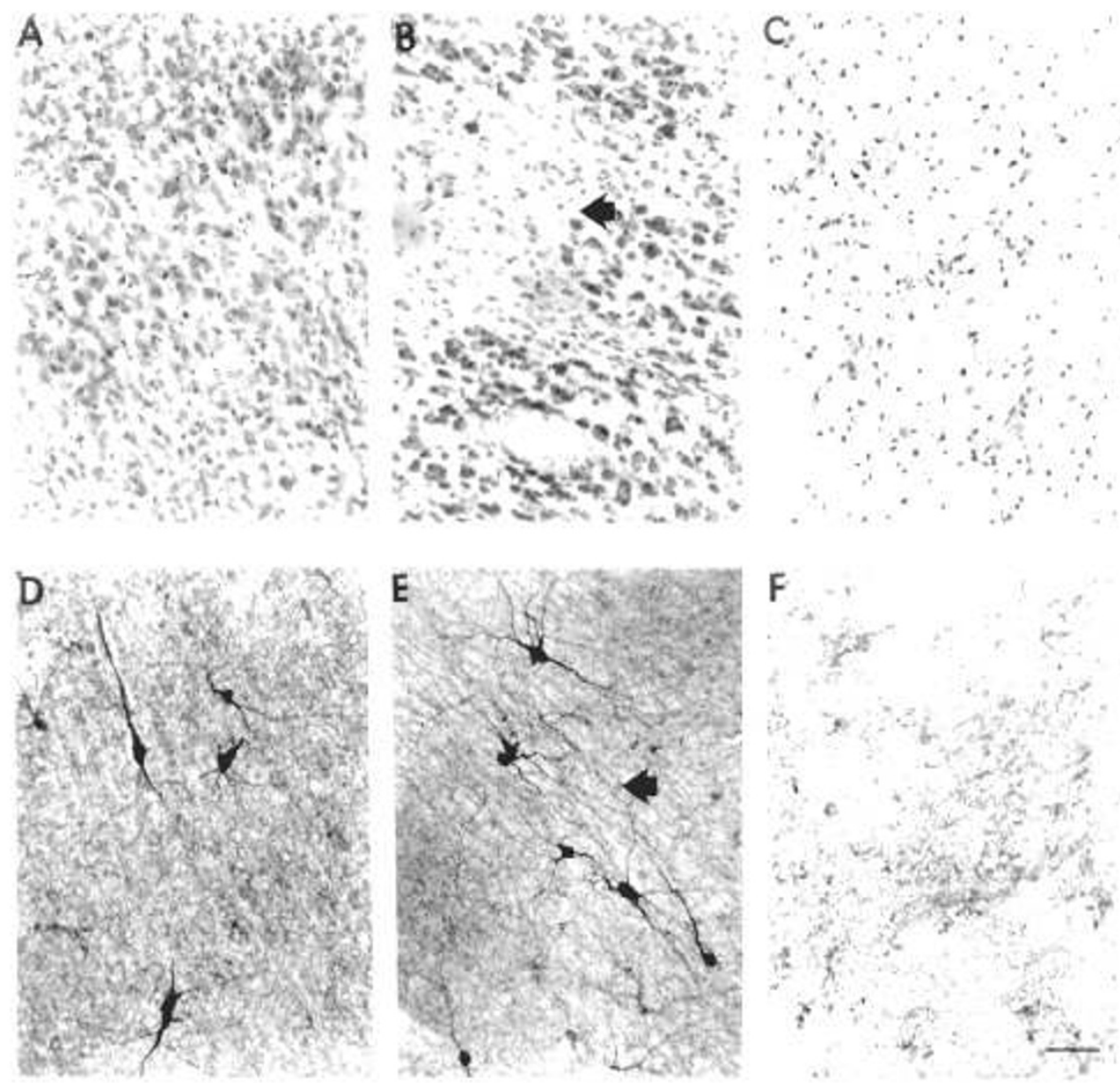

Fig. 4. Nissl-stained coronal section from a nonischemic, glucose-treated control brain shows normal cellular morphology through the deep layers of the cortex $(A)$. Normoglycemic ischemic brain shows loss of neurons in an area of columnar cell loss (arrow) $(B)$. Hyperglycemic ischemic brain shows complete loss of neurons with glial cells remaining $(C)$. NADPH-d reactive cells in a nonischemic, glucose-treated brain are scattered throughout the deep layers of the cortex $(D)$. In the area of infarction seen in $B($ arrow), there is preservation of the NADPH-d reactive neurons $(E)$. An alternate section from the brain shown in $C$ demonstrates loss of the NADPH-d reactive cells $(F)$. Scale bar $=50 \mu \mathrm{m}$.

sive $(16,18)$ and may result in activation of different receptormediated injury cascades than those seen in focal ischemia, such as that produced by the Levine procedure (19). Our results demonstrate that glucose administration after prolonged hypoxic exposure does not protect the neonatal brain from focal hypoxicischemic injury and, indeed, can exacerbate the severity of brain damage after hypoxia-ischemia.

The timing and extent of the ischemic insult in relation to the presence of systemic hyperglycemia may be more important determinants of neurologic compromise. Glucose given before global ischemia in adult rats causes augmentation of morphologic brain damage (9). In a forebrain ischemia model in adult rats preloaded with glucose, postischemic treatment with low-dose insulin reduced mortality and the incidence of postischemic seizures (11). However, a recent study in neonatal rat showed an increase in mortality when animals were insulin-treated before induction of hypoxia-ischemia (20). Juvenile monkeys who received infusions of glucose just before cardiac arrest showed widespread cortical and basal ganglia necrosis (8). However, in the neonatal rat, glucose infusion before induction of hypoxiaischemia caused no more brain damage than did hypoxia-ischemia alone (12). In the neonatal brain, hyperglycemia before the induction of hypoxia-ischemia improves glucose transport into brain but does not lead to enhanced glucose utilization or lactate accumulation by the immature brain (13).

Differential effects of glucose on neurons of varying maturity may also explain the differences in the effects of hyperglycemia in both human and animal models (21). If the mechanism of injury in hypoxia-ischemia is mediated through EAA receptors, as has been suggested by numerous investigators (22), then the discrepancy in the response to hyperglycemia after ischemia in different age groups might be explained by the ontogeny of the EAA receptors. In rodents from postnatal d 1 to $8,85 \%$ of CA 3 hippocampal neurons exhibit N-methyl-D-aspartate receptor-regulated spontaneous depolarization potentials, whereas in postnatal 9-12 rats less than $50 \%$ of neurons exhibit this characteristic (23). In support of these animal data, it was recently reported that in children with near-drowning an initially elevated blood glucose level was highly predictive of death or of survival in a persistent vegetative state (24).

In postischemic cerebral tissue, a persistent hypermetabolism that uses anaerobic over oxidative metabolism has been seen during reperfusion in adult animals (25), with damage to mitochondria in the regions of ischemia (26). It is possible then that glucose supplementation may provide the necessary substrate for generation of EAA receptor activation in the presence of injured neurons, thus augmenting cerebral damage, as was seen in our glucose-treated animals.

The cellular mechanisms by which glucose might further exacerbate the degree of hypoxic-ischemic brain damage cannot be ascertained by our study because we did not measure brain concentrations of glucose or lactate. However, our findings suggest that the exacerbating effect of hyperglycemia after hypoxiaischemia is related to other intracellular metabolic alterations that occur after focal ischemia, such as heightened EAA receptor 
activation. The long periods of postischemic hypoxia used in our study may contribute to this activation.

Our results suggest a significant deleterious effect of hyperglycemia after hypoxia-ischemia in the neonatal rat rather than a protective effect of glucose in terms of survival after hypoxia (14). Because current standards of care for the resuscitation of asphyxiated newborn infants call for i.v. glucose infusions, physicians should monitor blood glucose levels frequently and should infuse glucose to maintain blood glucose levels within physiologic ranges, preventing both hypoglycemic and hyperglycemic contributions to compromised neuronal survival.

\section{REFERENCES}

1. Hill A 1991 Current concepts of hypoxic ischemic cerebral injury in the term newborn. Pediatr Neurol 7:317-325

2. Volpe JJ 1987 Neurology of the Newborn. WB Saunders, Philadelphia, pp 160-279

3. Pildes R. Forbes AE, O'Connor SM, Cornblath M 1967 The incidence of neonatal hypoglycemia: a completed survey. J Pediatr 70:76-80

4. Lubchenko LO. Bard HL 1971 Incidence of hypoglycemia in newborn infants classified by birth weight and gestational age. Pediatrics 47:831-838

5. Gutberlet RL, Cornblath M 1976 Neonatal hypoglycemia revisited: 1975. Pediatrics 58:10-17

6. Jacobs MM, Phibbs RH 1989 Prevention, recognition, and treatment of perinatal asphyxia. Clin Perinatol 16:785-807

7. Cremer JE 1982 Substrate utilization and brain development. J Cereb Blood Flow Metab 2:394-407

8. Myers RE, Yamaguchi S-I 1977 Nervous system effects of cardiac arrest in monkeys: preservation of vision. Arch Neurol 34:65-74

9. Pulsinelli WA, Waldman S, Rawlinson D, Plum F 1982 Moderate hyperglycemia augments ischemic brain damage: a neuropathologic study in the rat. Neurology 32:1239-1246

10. Kalimo H, Rehncrona S, Söderfelt B, Olsson Y, Siesjö BK 1981 Brain lactic acidosis and ischemic cell damage. 2. Histopathology. J Cereb Blood Flow Metab 1:313-327
11. Voll CL, Auer RN 1988 The effect of postischemic blood glucose levels on ischemic brain damage in the rat. Ann Neurol 24:638-646

12. Voorhies TM, Rawlinson D, Vannucci RC 1986 Glucose and perinatal hypoxic-ischemic brain damage in the rat. Neurology 36:1115-1118

13. Vannucci RC, Vasta F, Vannucci SJ 1987 Cerebral metabolic responses of hyperglycemic immature rats to hypoxia-ischemia. Pediatr Res 21:524-529

14. Hattori H, Wasterlain CG 1990 Posthypoxic glucose supplement reduces hypoxic-ischemic brain damage in the neonatal rat. Ann Neurol 28:122-128

15. Levine $S 1960$ Anoxic-ischemic encephalopathy in rats. Am J Pathol 36:1-17

16. Rice JE, Vannucci RC, Brierley JB 1981 The influence of immaturity on hypoxic-ischemic brain damage in the rat. Ann Neurol 9:131-141

17. Ferriero DM, Arcavi LJ, Sagar SM, McIntosh TK, Simon RP 1988 Selective sparing of NADPH-diaphorase neurons in neonatal hypoxia-ischemia. Ann Neurol 24:670-676

18. Vannucci RC. Lyons DT, Vasta F 1988 Regional cerebral blood flow during hypoxia-ischemia in immature rats. Stroke 19:245-250

19. Sheardon MJ, Nielsen EO, Hansen AJ, Jacobsen P, Honoré T 1990 2,3dihydroxy 6-nitro-7-sulfamoyl-benzo $(F)$ quinoxaline: a neuroprotectant for cerebral ischemia. Science 247:571-574

20. Yager JY, Heitjan DF, Towfighi J, Vannucci RC 1992 Effect of insulininduced and fasting hypoglycemia on perinatal hypoxic-ischemia brain damage. Pediatr Res 31:138-142

21. Vannucci RC, Yager JY 1992 Glucose, lactic acid, and perinatal hypoxicischemic brain damage. Pediatr Neurol 8:3-12

22. McDonald JW, Johnston MV 1990 Physiological and pathophysiological roles of excitatory amino acids during central nervous system development. Brain Res Rev 15:41-70

23. Ben-Ari Y. Cherubini E, Corradetti R, Gaiarsa J-L 1989 Giant synaptic potentials in immature rat CA3 hippocampal neurons. J Physiol (Lond) 416:303-325

24. Ashwal S, Schneider S. Tomasi L, Thompson J 1990 Prognostic implications of hyperglycemia and reduced cerebral blood flow in childhood near-drowning. Neurology 40:820-823

25. Nemoto EM, Hossman K-A, Cooper HK 1981 Post-ischemic hypermetabolism in cat brain. Stroke 12:666-676

26. Hillered L, Smith M-L, Siesjö BK 1985 Lactic acidosis and recovery of mitochondrial function following forebrain ischemia in the rat. $J$ Cereb Blood Flow Metab 5:259-266 